\title{
Autologous Cytotoxic T-lymphocytes Induced with MUC1 Peptide-pulsed Dendritic Cells
}

National Cancer Institute

\section{Source}

National Cancer Institute. Autologous Cytotoxic T-lymphocytes Induced with MUC1

Peptide-pulsed Dendritic Cells. NCI Thesaurus. Code C124998.

A preparation of autologous cytotoxic $T$-lymphocytes $(C T L)$, specifically reactive to the tumor-associated antigen (TAA) mucin-1 (MUC1), with potential antineoplastic activity. Peripheral blood mononuclear cells (PBMCs) are collected from the patient with MUC1positive tumors and are exposed ex vivo to dendritic cells (DCs) that are pulsed with a MUC1 peptide to generate MUC1-specific CT Ls, which are subsequently expanded in vitro. Upon re-infusion of autologous CT Ls induced with MUC1 peptide-pulsed DCs to the patient, the CT Ls target and lyse the MUC1-expressing tumor cells. This inhibits tumor cell proliferation. MUC1 is expressed by a variety of tumor cell types. 